\title{
Higher Order Crack-Tip Fields for Anti-plane Crack in Exponential Functionally Graded Piezoelectric Materials
}

\author{
Xiao Chong ${ }^{1}$, Yao Dai ${ }^{2}$, Dianhui Hou ${ }^{3}$,Junguo $\mathrm{Ma}^{4}$ \\ ${ }^{1}$ Changping School of Noncommissioned Officer, PLA Academy of Equipment, Beijing, 101416, \\ China \\ ${ }^{2}$ Academy of Armored Force Engineering, Beijing, 100072, China \\ ${ }^{3}$ PLA University of Science and Technology,Nanjing,210007 \\ ${ }^{4}$ Changping School of Noncommissioned Officer, PLA Academy of Equipment, Beijing, 101416, \\ China
}

Keywords: crack tip fields, FGPMs, eigen-expansion method, anti-plane crack.

Abstract:The near-tip fields of an anti-plane crack in functionally graded piezoelectric materials(FGPMs) are investigated. To make the analysis tractable as usual, the elastic stiffness, piezoelectric parameter, and dielectric permittivity of FGPMs are assumed to be exponential functions of $x$ parallel to the crack. The boundary conditions on crack surfaces are assumed to be the stress free and electrically impermeable. The high order crack tip stress and electric displacement fields are obtained by the eigen-expansion method. This study possesses fundamental significance as Williams' solution to homogeneous materials.

\section{Introduction}

To get rid of the possible interfacial failure, the functionally graded piezoelectric materials have been introduced to reduce the stress concentration at the interface. In order to make FGPMs to have high strength, high reliability and long lifetime, the behaviors of cracks in brittle piezoelectric materials have to be analyzed. Therefore, the fracture mechanics problems of FGPMs have received much attention in recent decades. Wang and Noda[1] firstly studied the thermo- piezoelectric fracture problem of a functionally graded piezoelectric layer bonded to a metal. Li and Weng[2] considered the static anti-plane problem of a finite crack in a functionally graded piezoelectric material strip. It is found that the singular behaviors of both stress and electric displacement are the same as those in a homogeneous piezoelectric material. Shin[3] examined the singular stresses and electric fields in a functionally graded piezoelectric ceramic strip containing an eccentric crack off the center line under anti-plane shear loading with the theory of linear piezoelectricity. Mousavi[4] investigated the fracture behavior of a cracked functionally graded piezoelectric strip under antiplane mechanical and in-plane electrical loading. It should be mentioned that most existing studies in fracture analysis of FGPMs are focused on the singular part of crack tip fields. No attempts have been made in giving the high order crack tip fields for FGPMs. In this paper, we extend the Williams' solution to fracture problem of FGPMs and the stress and electric displacement high order fields are obtained.

\section{Basic equations}

Consider a crack in a functionally graded piezoelectric materials under anti-plane shear tractions and in-plane electric displacements. The FGPM is poled in the $\mathrm{z}$ direction and isotropic in the xoy plane. The present work employs exponential function to describe the continuous variations of material properties,

$$
\begin{gathered}
c_{44}=c_{440} f(r, \theta), \quad e_{15}=e_{150} g(r, \theta), \quad \varepsilon_{11}=\varepsilon_{110} j(r, \theta) \\
f(r, \theta)=e^{\beta_{1} r \cos \theta}, \quad g(r, \theta)=e^{\beta_{2} r \cos \theta}, \quad j(r, \theta)=e^{\beta_{3} r \cos \theta}
\end{gathered}
$$

where $c_{440}$ is the shear modulus, $e_{150}$ is the piezoelectric coefficient, $\varepsilon_{110}$ is the dielectric parameter at $x=0$.

The governing equations can be written as 


$$
\left\{\begin{array}{l}
c_{44} \nabla^{2} w+\frac{\partial c_{44}}{\partial r} \frac{\partial w}{\partial r}+\frac{\partial c_{44}}{r^{2} \partial \theta} \frac{\partial w}{\partial \theta}+e_{15} \nabla^{2} \phi+\frac{\partial e_{15}}{\partial r} \frac{\partial \phi}{\partial r}+\frac{\partial e_{15}}{r^{2} \partial \theta} \frac{\partial \phi}{\partial \theta}=0 \\
e_{15} \nabla^{2} w+\frac{\partial e_{15}}{\partial r} \frac{\partial w}{\partial r}+\frac{\partial e_{15}}{r^{2} \partial \theta} \frac{\partial w}{\partial \theta}-\varepsilon_{11} \nabla^{2} \phi-\frac{\partial \varepsilon_{11}}{\partial r} \frac{\partial \phi}{\partial r}-\frac{\partial \varepsilon_{11}}{r^{2} \partial \theta} \frac{\partial \phi}{\partial \theta}=0
\end{array}\right.
$$

where $\nabla^{2}=\frac{\partial^{2}}{\partial r^{2}}+\frac{1}{r} \frac{\partial}{\partial r}+\frac{1}{r^{2}} \frac{\partial^{2}}{\partial \theta^{2}}$ is the two-dimensional Laplace operator.

\section{The higher order crack-tip field}

The displacement component $w$ and the electric potential $\phi$ can be expanded as follows

$$
w=\sum_{i=1}^{\infty} r^{\frac{i}{2}} w_{i}(\theta), \quad \phi=\sum_{i=1}^{\infty} r^{\frac{i}{2}} \phi_{i}(\theta)
$$

where, $w_{i}(\theta)$ and $\phi_{i}(\theta)$ are eigen-functions.

Substitute Eq. (4)into Eq.(3). According to the linear independence of $r^{-3 / 2}, r^{-1}, r^{-1 / 2}, \ldots, r^{i / 2-2}, \ldots$, the system of ordinary differential equations are obtained.

In the case of electrically impermeable crack, the crack surfaces are free of electric charges and the electric displacement inside the crack is zero. As the crack surface is free, the boundary conditions are

Further, they can be expressed as

$$
\left.\sigma_{z y}\right|_{\theta= \pm \pi}=0,\left.\quad D_{y}\right|_{\theta= \pm \pi}=0
$$

$$
w_{i}^{\prime}( \pm \pi)=0 \quad \phi_{i}^{\prime}( \pm \pi)=0
$$

Solving the system of ordinary differential equations, we can obtain the results

$$
\begin{gathered}
\left\{\begin{array}{l}
w_{1}(\theta)=B_{11} \sin \frac{\theta}{2} \\
\phi_{1}(\theta)=B_{12} \sin \frac{\theta}{2}
\end{array}\right. \\
\left\{\begin{array}{l}
w_{2}(\theta)=B_{21} \cos \theta \\
\phi_{2}(\theta)=B_{22} \cos \theta
\end{array}\right. \\
\left\{\begin{array}{l}
w_{3}(\theta)=B_{31} \sin \frac{3 \theta}{2}+\frac{\left[B_{12}\left(\beta_{2}-\beta_{3}\right) e_{150}+c_{440} B_{11} \beta_{1}\right] \varepsilon_{110}+e_{150}^{2} \beta_{2} B_{11}}{4\left(e_{150}^{2}+\varepsilon_{110} c_{440}\right)} \sin \frac{\theta}{2} \\
\phi_{3}(\theta)=B_{32} \sin \frac{3 \theta}{2}-\frac{\left[B_{11}\left(\beta_{2}-\beta_{1}\right) e_{150}-\varepsilon_{110} \beta_{3} B_{12}\right] c_{440}-e_{150}{ }^{2} \beta_{2} B_{12}}{4\left(e_{150}^{2}+\varepsilon_{110} c_{440}\right)} \sin \frac{\theta}{2}
\end{array}\right. \\
\left\{\begin{array}{l}
w_{4}(\theta)=B_{41} \cos 2 \theta+\frac{\left[B_{22}\left(\beta_{3}-\beta_{2}\right) e_{150}-B_{21} \beta_{1} c_{440}\right] \varepsilon_{110}-e_{150}^{2} \beta_{2} B_{21}}{4\left(e_{150}^{2}+\varepsilon_{110} c_{440}\right)} \\
\phi_{4}(\theta)=B_{42} \cos 2 \theta-\frac{\left[\beta_{2} B_{22} e_{150}+B_{21} c_{440}\left(\beta_{1}-\beta_{2}\right)\right] e_{150}+\beta_{3} \varepsilon_{110} c_{440} B_{22}}{4\left(e_{150}^{2}+\varepsilon_{110} c_{440}\right)}
\end{array}\right.
\end{gathered}
$$




$$
\begin{aligned}
w_{5}(\theta)= & B_{51} \sin \frac{5 \theta}{2}+\frac{1}{24\left(e_{150}^{2}+\varepsilon_{110} c_{440}\right)^{2}}\left\{\left[\left(e_{150}^{4} \beta_{2}{ }^{2}-3 c_{440} e_{150}^{2} \varepsilon_{110}\right) \beta_{2}{ }^{2}+\left(c_{440}^{2} \varepsilon_{110}^{2}-\right.\right.\right. \\
& \left.\left.c_{440} e_{150}^{2} \varepsilon_{110}\right) \beta_{1}^{2}+2 c_{440} e_{150}^{2} \varepsilon_{110}\left(3 \beta_{1} \beta_{2}+\beta_{2} \beta_{3}-\beta_{1} \beta_{3}\right)\right] B_{11}+\left[\left(3 \varepsilon_{110} e_{150}^{3}-\right.\right. \\
& \left.c_{440} e_{150} \varepsilon_{110}^{2}\right) \beta_{2}{ }^{2}+\left(\varepsilon_{110} e_{150}^{3}-c_{440} e_{150} \varepsilon_{110}^{2}\right) \beta_{3}{ }^{2}-4 e_{150}^{3} \varepsilon_{110} \beta_{2} \beta_{3}+2 c_{440} e_{150} \varepsilon_{110}^{2} . \\
& \left.\left(\beta_{1} \beta_{2}+\beta_{2} \beta_{3}-\beta_{1} \beta_{3}\right)\right] B_{12}-3\left[\left(c_{440} e_{150}^{2} \varepsilon_{110}+e_{150}^{4}\right) \beta_{2}+\left(c_{440}^{2} \varepsilon_{110}^{2}+c_{440} e_{150}^{2} .\right.\right. \\
& \left.\left.\left.\varepsilon_{110}\right) \beta_{1}\right] B_{31}+3\left[\left(e_{150}^{3} \varepsilon_{110}+c_{440} e_{150} \varepsilon_{110}^{2}\right) \beta_{3}-\left(e_{150}^{3} \varepsilon_{110}+c_{440} e_{150} \varepsilon_{110}^{2}\right) \beta_{2}\right] B_{32}\right\} . \\
& \sin \frac{\theta}{2}+\frac{1}{32\left(e_{150}^{2}+\varepsilon_{110} c_{440}\right)^{2}}\left\{\left[\left(5 c_{440} e_{150}^{2} \varepsilon_{110}-e_{150}^{4}\right) \beta_{2}{ }^{2}+\left(2 c_{440} e_{150}^{2} \varepsilon_{110}-\right.\right.\right. \\
& \left.\left.c_{440}^{2} \varepsilon_{110}^{2}\right) \beta_{1}{ }^{2}-3 c_{440} e_{150}^{2} \varepsilon_{110}\left(3 \beta_{1} \beta_{2}+\beta_{2} \beta_{3}-\beta_{1} \beta_{3}\right)\right] B_{11}+\left[\left(2 c_{440} e_{150} \varepsilon_{110}^{2}-\right.\right. \\
& \left.4 e_{150}^{3} \varepsilon_{110}\right) \beta_{2}{ }^{2}+\left(c_{440} e_{150} \varepsilon_{110}^{2}-2 e_{150}^{3} \varepsilon_{110}\right) \beta_{3}{ }^{2}+6 e_{150}^{3} \varepsilon_{110} \beta_{2} \beta_{3}-3 c_{440} e_{150} \varepsilon_{110}^{2} . \\
& \left.\left.\left(\beta_{1} \beta_{2}+\beta_{2} \beta_{3}-\beta_{1} \beta_{3}\right)\right] B_{12}\right\} \sin \frac{3 \theta}{2}
\end{aligned}
$$

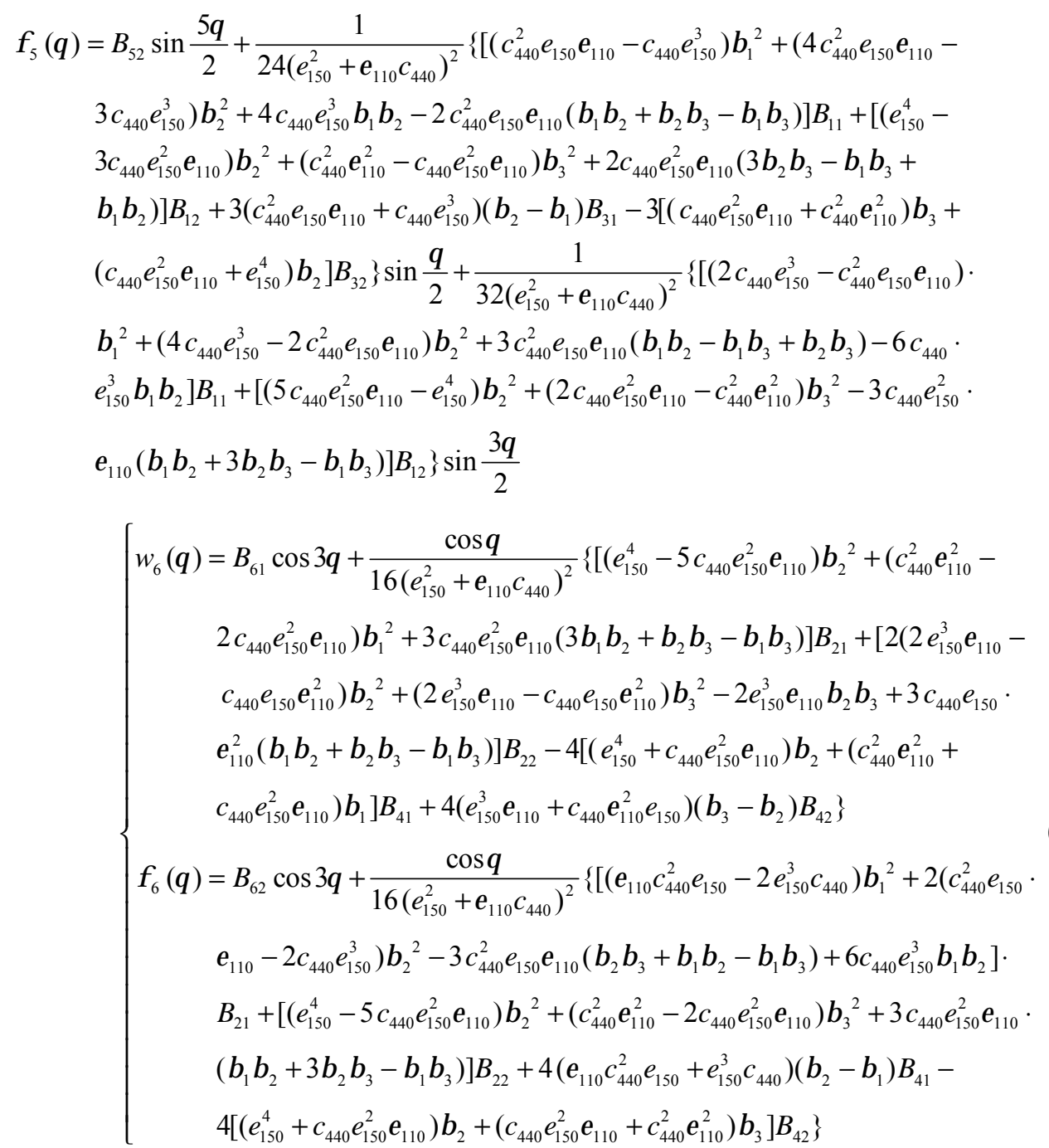

where $\mathbf{A}_{i j}=\left[\begin{array}{l}A_{i j} \\ B_{i j}\end{array}\right]$ are the undetermined coefficients.

Substituting Eq.(7)-(12) into Eq.(4), the displacement component $w$ and the electric potential $\phi$ are obtained.

Then , the stress and the electric displacement components can be obtained 


$$
\begin{gathered}
\tau_{x z}=c_{44} w_{, x}+e_{15} \phi_{, x}, \tau_{y z}=c_{44} w_{, y}+e_{15} \phi_{, y} \\
D_{x}=e_{15} w_{, x}-\varepsilon_{11} \phi_{, x}, D_{y}=e_{15} w_{, y}-\varepsilon_{11} \phi_{, y}
\end{gathered}
$$

The mode III stress intensity factor (SIF) and electric displacement intensity factor (EDIF) of the crack tip are defined as

$$
\left\{\begin{array}{l}
K^{T}=\lim _{r \rightarrow 0} \sqrt{2 \pi r} \sigma_{y z}(r, 0)=\frac{\sqrt{2 \pi}}{2}\left(c_{440} A_{11}-e_{150} B_{11}\right) \\
K^{D}=\lim _{r \rightarrow 0} \sqrt{2 \pi r} D_{y}(r, 0)=\frac{\sqrt{2 \pi}}{2}\left(e_{150} A_{11}+\varepsilon_{110} B_{11}\right)
\end{array}\right.
$$

\section{Conclusions}

The higher order displacement, electric potential, stress and electric displacement fields for exponential FGPMs are obtained in this paper. The effect of non-homogeneity reflects only in the higher order items. Due to coupling effect of piezoelectric material, the stress intensity factor and electric displacement intensity factor are depending on both displacement component and the electric potential. It is obvious that the crack tip fields and intensity factors obtained herein provide basic solutions to fracture problems of FGPMs in experimental analysis and engineering practice.

\section{Acknowledgements}

The research is supported by the National Natural Science Foundation of China (No11172332.)

\section{References}

[1] B.L. Wang \& N. Noda. Thermally induced fracture of a smart functionally graded composite structure. Theoretical and Applied Fracture Mechanics. 35, pp. 93-109, 2001.

[2] C. Li \& G.J. Weng. Antiplane crack problem in functionally graded piezoelectric materials. Journal of Applied Mechanics-Transactions of the Asme. 69, pp.481-488, 2002.

[3] J.W. Shin \& T.U. Kim. Functionally graded piezoelectric strip with eccentric crack under antiplane shear. KSME International Journal. 17, pp. 854-859, 2003.

[4] S.M. Mousavi \& J. Paavola. Analysis of cracked functionally graded piezoelectric strip. International Journal of Solids and Structures. 50, pp. 2449-2456, 2013. 